\title{
A Numerical Calculation of Arbitrary Integrals of Functions
}

\author{
Ojima John Mamman*, Terhemen Aboiyar
}

College of Science, Federal University of Agriculture, Makurdi

\section{* Corresponding Author email: mammanojima@gmail.com}

Article Histary

Received: 12 August 2019

Revised: 30 September 2019

Accepted: 03 October 2019

Published: 20 October 2019

Student(s)

- Ojima John Mamman

Academic Year: 2018-19

Course Level: Master

Course Name: Industrial Mathematics

Course year: Final Year

Mentar(s)

- $\quad$ Terhemen Aboiyar

\begin{abstract}
A BSTRACT
This paper presents a numerical technique for solving fractional integrals of functions by employing the trapezoidal rule in conjunction with the finite difference scheme. The proposed scheme is only a simple modification of the trapezoidal rule, in which it is treated as an algorithm in a sequence of small intervals for finding accurate approximate solutions to the corresponding problems. This method was applied to solve fractional integral of arbitrary order $\alpha>0$ for various values of alpha. The fractional integrals are described in the Riemann-Liouville sense. Figurative comparisons and error analysis between the exact value, twopoint and three-point central difference formulae reveal that this modified method is active and convenient.
\end{abstract}

Keywords: Finite difference, Integrals functions, fractional calculus, fractional integral, modified trapezoidal rule, Riemann-Liouville.

\section{Introduction}

This paper presents an approach to fractional integration which aims at introducing an algorithm for solving integrals of non-integer order. Fractional integration is a branch of fractional calculus that investigates integrals of arbitrary real and complex order with their applications. Since the emergence of the idea of arbitrary integrals they have been lack of physical and geometric interpretation of this operator which many authors have attempted to provide, for this reason much efforts have been devoted to overcoming this challenge [1]. Methods are being developed to provide tools for solving such problems, by using the Riemann-Liouville approach it is convenient to approximate the fractional integral using generalized and modified trapezoidal rule.

In recent times, large number of scientific and engineering problems involves fractional calculus, the reason for this is that it provides more accurate models of systems under consideration, a praiseworthy attempt has been given by podlubny and others to develop interpretation of fractional integration and differentiation [1] - [4]. For instance, fractional calculus is applied to model the dynamics of interfaces between nanoparticles and substrates [5], economics [6], frequency dependent damping behavior of many viscoelastic materials [7], signal processing [8], continuum and statistical mechanics [9], and oscillation [10]. 
In recent years considerable interest in fractional differential equation has been stimulated by the applications that it finds in the field of science, including numerical analysis, engineering, economics, biology, oil industry, finance and others [11] - [20]. Real life modeling of phenomenon having dependence not solely at the time instant, however additionally the previous time history can be successfully achieved by fractional calculus. Mathematical formulations of mentioned phenomena contain equations with fractional order, most of these kinds of equations are difficult to solve [21] and in some cases do not have exact analytic solutions, so approximation and numerical techniques must be used. For practical purposes however, analytical solutions are often insufficient. The history [22], challenges [23], methods [24] - [32] and progress [33] made in the field of fractional calculus are evident in many publications. The technique considered here can be utilized to approximate arbitrary integrals. The method given here can be used to compute such an approximation. This paper deals with the rationality of Modified Trapezoidal rule in conjunction with the finite difference scheme for solving fractional integral. Given that the interval $[a, b]$ is subdivided into $M$ subintervals $\left[x_{k}, x_{k+1}\right]$ of width $h=\frac{(b-a)}{M}$ by using the equally spaced nodes $x_{k}=a+$ $k h$ for $k=0,1, \ldots, M$. The composite trapezoidal rule for $\mathrm{M}$ subintervals can be expressed in any of three equivalent ways:

$\mathrm{T}(f, h)=\frac{h}{2} \sum_{k=1}^{M}\left(f\left(x_{k-1}\right)+f\left(x_{k}\right)\right.$

or

$T(f, h)=\frac{h}{2}\left(f_{0}+2 f_{1}+2 f_{2}+2 f_{3}+\ldots+2 f_{m-2}+2 f_{m-1}+f_{m}\right)$

or

$\mathrm{T}(f, h)=\frac{h}{2}(f(a)+f(b))+h \sum_{k=1}^{M-1} f\left(x_{k}\right)$.

This is an approximation to the integral of $f(x)$ over $[\mathrm{a}, \mathrm{b}]$, and we write

$\int_{a}^{b} f(x) d x \approx T(f, h)$.

\subsection{Error Analysis}

If $f(x) \in C^{2}[x, y]$, then there is a value $\tau$ with $x<\tau<y$ so that the error term $E(f, h)$ has the form

$$
E(f, h)=\frac{-(y-x) f^{(2)}(\tau)\left(h^{2}\right)}{12}=O\left(h^{2}\right)
$$

In cases where the derivatives of $f(x)$ is known, the formula can be used to estimate the number of subintervals required to achieve a specified accuracy.

where

$E(f, h)=\int_{a}^{b} f(x) d x-T(f, h)$.

\section{Materials and Techniques}

In this area, we give some important definitions and properties of fractional calculus theory [34] and [35] which will be utilized further in this paper.

Definitions 2.1: The Riemann-Liouville fractional integral operator $J^{\alpha} f(x)$ of order $\alpha>0$ on the usual Lebesgue space $L_{1}[a, b]$ is given by

$J^{\alpha} f(x)=\frac{1}{\Gamma(\alpha)} \int_{0}^{x}(x-\tau)^{\alpha-1} f(\tau) d \tau, x>0, \alpha>0$.

Properties of the operator $J^{\alpha}$ and details are given below:

For $\alpha, \beta, x>0$ and $\lambda>-1$, we have

(i) $J^{\alpha} J^{\beta}=J^{\alpha+\beta}$,

(ii) $J^{\alpha} x^{\lambda}=\frac{\Gamma(\lambda+1)}{\Gamma(\alpha+\lambda+1)} x^{\alpha+\lambda}$,

(iii) $J^{\alpha} \cos (a x)=x^{\alpha} \sum_{k=0}^{\infty} \frac{(-1)^{k}(a x)^{2 k}}{\Gamma(\alpha+2 k+1)}$, 
(iv) $J^{\alpha} \sin (a x)=x^{\alpha} \sum_{k=0}^{\infty} \frac{(-1)^{k}(a x)^{2 k+1}}{\Gamma(\alpha+2 k+2)}$,

(v) $J^{\alpha} e^{a x}=x^{\alpha} \sum_{k=0}^{\infty} \frac{(a x)^{k}}{\Gamma(\alpha+k+1)}$.

Theorem 1: [34] suppose that $f \in \boldsymbol{C}^{2}[0, T], \tilde{f}_{k}$ is the piecewise linear interpolation for $f$ with nodes chosen at the $t_{j}=j h$ with $h=\frac{T}{k}, j=0,1,2, \ldots, k$, then

(1) $\int_{0}^{t_{k}}\left(t_{k}-t\right)^{\alpha-1} f_{k}(t) d t=\sum_{j=0}^{k} a_{j, k} \cdot f\left(t_{i}\right)$,

where

$a_{j, k}=\frac{h^{\alpha}}{\alpha(\alpha+1)}\left\{\begin{array}{lc}(k-1)^{\alpha+1}-(k-1-\alpha) k^{\alpha}, & j=0, \\ (k-j+1)^{\alpha+1}+(k-j-1)^{\alpha+1}-2(k-j)^{\alpha+1}, 1 \leq j \leq k-1,(14) & j=k,\end{array}\right.$

(ii) $\left|\int_{0}^{t_{k}}\left(t_{k}-t\right)^{\alpha-1} f(t) d t-\sum_{j=0}^{k} a_{j, k} \cdot f\left(t_{i}\right)\right| \leq C_{\alpha}\left\|f^{\prime \prime}\right\|_{\infty} t_{k}^{\alpha} h^{2}$,

for some constant depending on $\alpha$.

\subsection{The Trapezoidal Rule}

A spontaneous approach of finding the area under the curve $y=f(x)$ over $[a, b]$ is by approximating that area with a series of trapezoids that lie above the intervals

$\left\{\left[x_{k}, x_{k+1}\right]\right\}$. Here, we consider a generalized form of trapezoidal rule to approximate the fractional integral $J^{\alpha} f(x)$ of order $\alpha>0$ by a weighted sum of function values at specified points.

Theorem 2: [35] Given that the interval $[0, a]$ is subdivided into k subintervals $\left[x_{j}, x_{j+1}\right]$ of equal width $h=a / k$ by using the nodes $x_{\mathrm{j}}=j h$, for $\mathrm{j}=0,1, \ldots, \mathrm{k}$. The modified trapezoidal rule is given as:

$T(f, h, \alpha)=\left((k-1)^{\alpha+1}-(\mathrm{k}-\alpha-1) \mathrm{k}^{\alpha}\right) \frac{h^{\alpha} f(0)}{\Gamma(\alpha+2)}+\frac{h^{\alpha} f(a)}{\Gamma(\alpha+2)}$

$+\sum_{j=1}^{k-1}\left((\mathrm{k}-\mathrm{j}+1)^{\alpha+1}-2(\mathrm{k}-\mathrm{j})^{\alpha+1}+(\mathrm{k}-\mathrm{j}-1)^{\alpha+1}\right) \frac{h^{\alpha} f\left(x_{j}\right)}{\Gamma(\alpha+2)}$

this gives an approximation to fractional integral

$\left(J^{\alpha} f(x)\right)(a)=T(f, h, \alpha)-E_{T}(f, h, \alpha), \quad a>0, \quad \alpha>0$.

Proof: From definition (7) we have

$\left(J^{\alpha} f(x)\right)(a)=\frac{1}{\Gamma(\alpha)} \int_{0}^{a}(a-\tau)^{\alpha-1} f(\tau) d \tau$.

If $f_{k}$ is the piecewise linear interpolant for $f$ whose nodes are chosen at the nodes $x_{j}, j=0,1,2, \ldots, k$, then, we have

$\int_{0}^{a}(a-\tau)^{\alpha-1} f_{k}(\tau) d \tau=\frac{\boldsymbol{h}^{\alpha}}{\boldsymbol{\alpha}(\boldsymbol{\alpha}+1)} \cdot\left\{\begin{array}{c}\left((k-1)^{\alpha+1}-(k-\alpha-1) k^{\alpha}\right) f(0)+f(a) \\ +\sum_{j=1}^{k-1}\left((k-j+1)^{\alpha+1}-2(k-j)^{\alpha+1}+(k-j-1)^{\alpha+1}\right) f\left(x_{j}\right)\end{array}\right.$

and

$\left|\int_{0}^{a}(a-\tau)^{\alpha-1} f(\tau)-\int_{0}^{a}(a-\tau)^{\alpha-1} \tilde{f}_{k}(\tau) d \tau\right| \leq C_{\alpha}\left\|f^{\prime \prime}\right\|_{\infty} a^{\alpha} h^{2}$.

from (19) and (20) we get Theorem 2, where $C_{\alpha}^{\prime}=C_{\alpha} / \Gamma(\alpha)$.

If $\alpha=1$ the modified trapezoidal rule reduces to the classical trapezoidal rule (3). This method behaves in a way that is comparable to the classical trapezoidal rule. It is well established that, for small $h$, using the central-difference formulas [36], [37] and [38] we can approximate the integral. The two-point and threepoint central difference formula for $f\left(x_{j}\right)$ are given as:

$f\left(x_{j}\right)=\frac{f\left(x_{j}+h\right)+f\left(x_{j}-h\right)}{2}+O(h)$ 
A Numerical Calculation of Arbitrary Integrals of Functions

$f\left(x_{j}\right)=\frac{f\left(x_{j}+2 h\right)+f\left(x_{j}-2 h\right)}{2}+O(h)$

This can also be extended to give

$f\left(x_{j}\right)=\frac{f\left(x_{j}+n h\right)+f\left(x_{j}-n h\right)}{2}+O(h)$

where $n$ is any real number.

\section{Theory/calculation}

Example 1.: Approximate the fractional integral of the function $f(x)=\cos x$, given that $\alpha$ is $=\frac{3}{4}$

Table 1: The modified trapezoidal rule for $\left(J^{0.75} \cos x\right)(1)$

\begin{tabular}{|l|l|l|l|l|l|l|}
\hline $\mathrm{K}$ & $\mathrm{h}$ & Exact Value & $\begin{array}{l}\boldsymbol{T}(\boldsymbol{f}, \boldsymbol{h} . \mathbf{0 . 7 5}) \text { at } \\
2 \text { point cent. diff. }\end{array}$ & $\boldsymbol{T}_{\boldsymbol{T}}(\boldsymbol{f}, \boldsymbol{h}, \mathbf{0 . 7 5})$ & $\begin{array}{l}\boldsymbol{T}(\boldsymbol{f}, \boldsymbol{h}, \mathbf{0 . 7 5}) \text { at } \\
3 \text { point cent. diff. }\end{array}$ & $\boldsymbol{T}_{T}(\boldsymbol{f}, \boldsymbol{h}, \mathbf{0 . 7 5})$ \\
\hline $\mathbf{1 0}$ & 0.1 & 0.874344373 & 0.869256566 & 0.005087807 & 0.856206776 & 0.018137597 \\
\hline $\mathbf{2 0}$ & 0.05 & 0.874344373 & 0.873070571 & 0.001273802 & 0.869795873 & 0.004548500 \\
\hline $\mathbf{4 0}$ & 0.025 & 0.874344373 & 0.874025757 & 0.000318616 & 0.873206315 & 0.001138058 \\
\hline $\mathbf{8 0}$ & 0.0125 & 0.874344373 & 0.874264701 & 0.000079672 & 0.874059793 & 0.000284580 \\
\hline $\mathbf{1 6 0}$ & 0.00625 & 0.874344373 & 0.874324453 & 0.000019920 & 0.874273223 & 0.000071150 \\
\hline $\mathbf{3 2 0}$ & 0.003125 & 0.874344373 & 0.874339393 & 0.000004980 & 0.874326585 & 0.000017788 \\
\hline
\end{tabular}

Tables 1 gives the approximated values for the fractional integral $\left(J^{\alpha} f(x)\right)(1)$ when $\alpha$ is $=\frac{3}{4}$ using the modified trapezoidal rule (16). With the exact solution which is the true value of the fractional integral given as (10).

Example 2.: Solve the function $f(x)=\cos x$, when $\alpha$ is $=1$.

Table 2: The modified trapezoidal rule for $\left(J^{1} \cos x\right)(1)$

\begin{tabular}{|l|l|l|l|l|l|l|}
\hline $\mathrm{K}$ & $\mathrm{h}$ & Exact Value & $\begin{array}{l}\boldsymbol{T}(\boldsymbol{f}, \boldsymbol{h}, \mathbf{1}) \text { at } 2 \\
\text { point cent. diff. }\end{array}$ & $\boldsymbol{T}_{\boldsymbol{T}}(\boldsymbol{f}, \boldsymbol{h}, \mathbf{1})$ & $\begin{array}{l}\boldsymbol{T}(\boldsymbol{f}, \boldsymbol{h}, \mathbf{1}) \text { at } 3 \\
\text { point cent. diff. }\end{array}$ & $\boldsymbol{T}_{\boldsymbol{T}}(\boldsymbol{f}, \boldsymbol{h}, \mathbf{1})$ \\
\hline $\mathbf{1 0}$ & 0.1 & 0.841470985 & 0.836569296 & 0.004901689 & 0.824010226 & 0.017460759 \\
\hline $\mathbf{2 0}$ & 0.05 & 0.841470985 & 0.840244271 & 0.001226714 & 0.837092697 & 0.004378288 \\
\hline $\mathbf{4 0}$ & 0.025 & 0.841470985 & 0.841164225 & 0.000306759 & 0.840375593 & 0.001095392 \\
\hline $\mathbf{8 0}$ & 0.0125 & 0.841470985 & 0.841394290 & 0.000076695 & 0.841197086 & 0.000273899 \\
\hline $\mathbf{1 6 0}$ & 0.00625 & 0.841470985 & 0.841451811 & 0.000019174 & 0.8414025068 & 0.000068478 \\
\hline $\mathbf{3 2 0}$ & 0.003125 & 0.841470985 & 0.841466191 & 0.000004794 & 0.841453865 & 0.000017120 \\
\hline
\end{tabular}

Tables 2.: This gives the approximated values for the fractional integral $\left(J^{\alpha} f(x)\right)(1)$ using (16) the modified trapezoidal rule and (10) gives the exact solution which is the true value of the fractional integral. 
Example 3.: Approximate the function $f(x)=\cos x$, when $\alpha$ is $=1.25$

Table 3: The modified trapezoidal rule for $\left(J^{1.25} \cos x\right)(1)$

\begin{tabular}{|l|l|l|l|l|l|l|}
\hline $\mathrm{K}$ & $\mathrm{h}$ & Exact Value & $\begin{array}{l}\boldsymbol{T}(f, \boldsymbol{h}, \mathbf{1 . 2 5}) \text { at } \\
2 \text { point cent. diff. }\end{array}$ & $\boldsymbol{T}_{T}(f, \boldsymbol{h}, \mathbf{1 . 2 5})$ & $\begin{array}{l}\boldsymbol{T}(f, \boldsymbol{h}, \mathbf{1 . 2 5}) \text { at } \\
3 \text { point cent. } \\
\text { Diff }\end{array}$ & $\boldsymbol{T}_{T}(f, \boldsymbol{h}, \mathbf{1 . 2 5})$ \\
\hline $\mathbf{1 0}$ & 0.1 & 0.767202941 & 0.762733425 & 0.004469516 & 0.751282822 & 0.015920119 \\
\hline $\mathbf{2 0}$ & 0.05 & 0.767202941 & 0.766084441 & 0.001118501 & 0.763211024 & 0.003991917 \\
\hline $\mathbf{4 0}$ & 0.025 & 0.767202941 & 0.766923250 & 0.000279691 & 0.766204222 & 0.000998719 \\
\hline $\mathbf{8 0}$ & 0.0125 & 0.767202941 & 0.767133015 & 0.000069927 & 0.766953216 & 0.000249726 \\
\hline $\mathbf{1 6 0}$ & 0.00625 & 0.767202941 & 0.767185460 & 0.000017482 & 0.767140507 & 0.000062434 \\
\hline $\mathbf{3 2 0}$ & 0.003125 & 0.767202941 & 0.767198571 & 0.000004370 & 0.767187333 & 0.000015609 \\
\hline
\end{tabular}

Tables 3.: gives the approximated values for the fractional integral $\left(J^{\alpha} f(x)\right)(1)$ when $\alpha$ is $=1.25$ using the modified trapezoidal rule (16). With the exact solution which is the true value of the fractional integral given as (10).

\section{Results and Discussion}

In example one, we solved the function $f(x)=\cos x$ when $\alpha$ is $=\frac{3}{4}$ and then used the modified trapezoidal rule in conjunction with the two-step and three-step central difference formula to approximate the fractional integral when $\alpha$ is $=\frac{3}{4}$. Table 1 shows the numerical values and errors when compared with the exact solution and figure 1 gives the exact and approximate solutions of example 1 for table 1 at alpha $=\frac{3}{4}$ and how it converges to the exact solution as the step size decreases while figure 2 shows the absolute error function of example 1 for Table 1 at alpha $=\frac{3}{4}$. Similarly, the second and third example evaluates the integral function $f(x)=\cos x$ at $\alpha=1.0$ and 1.25 respectively.

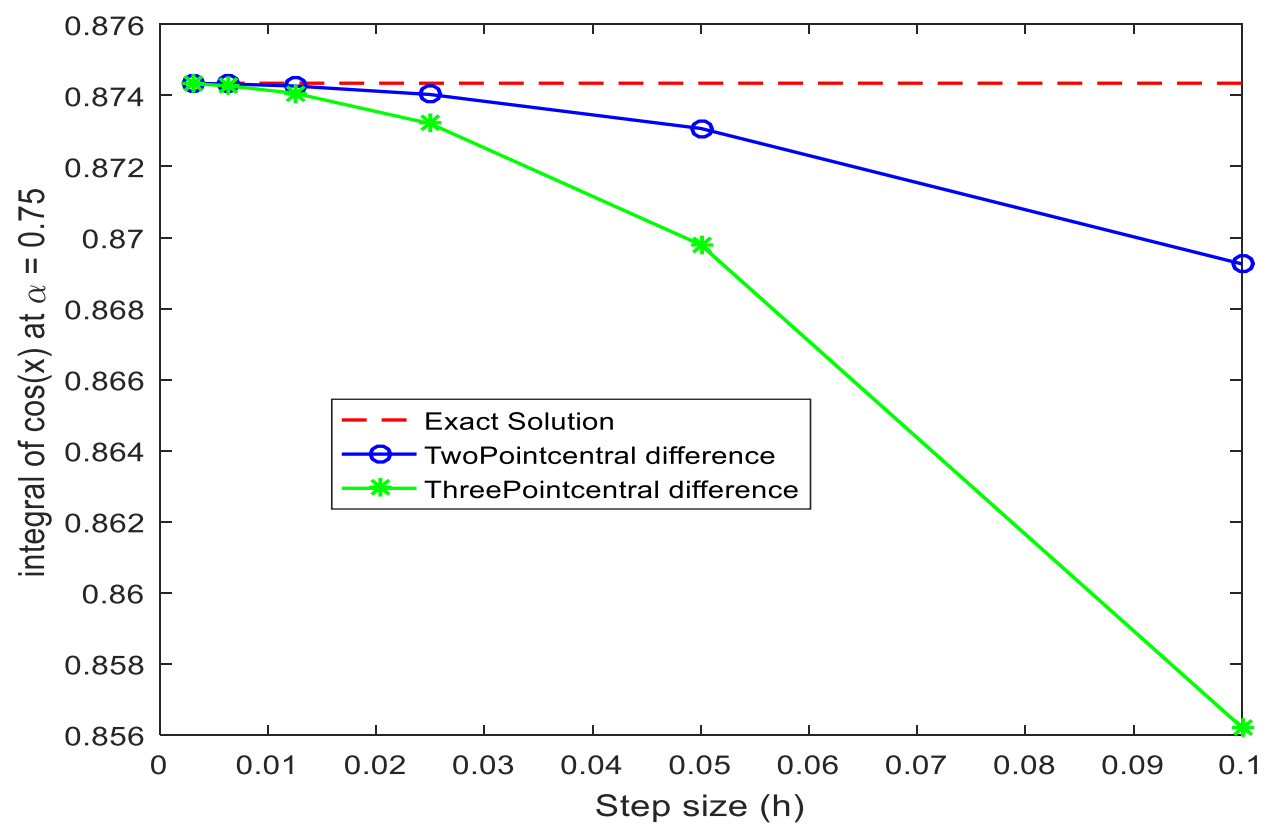

Figure 1: Exact and approximate solutions of example 1 for table 1 at alpha $=\frac{3}{4}$. 


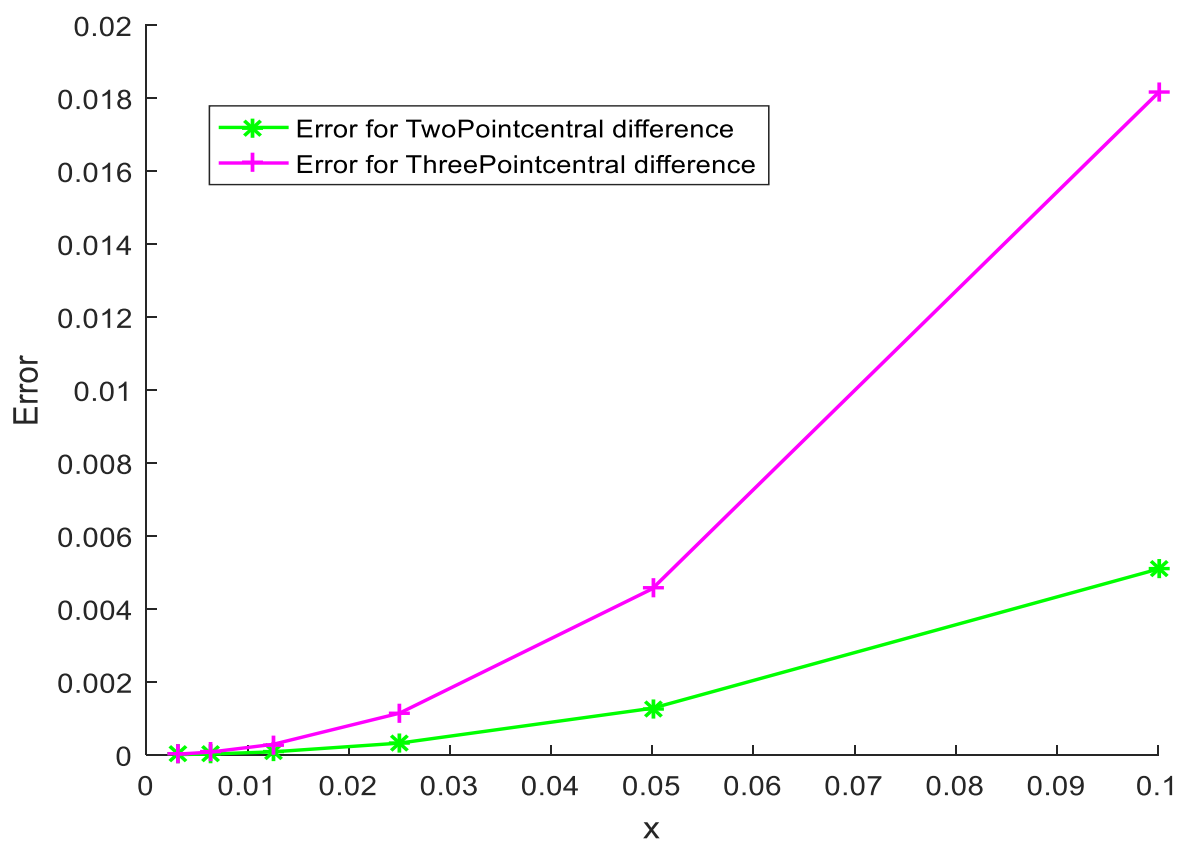

Figure 2: Absolute error function of example 1 for Table 1 at alpha $=\frac{3}{4}$.

\section{Conclusion}

We have successfully applied the modified trapezoidal rule to approximate fractional integrals of functions, in each case we try to solve problems for which the analytic solution is available. This approach gives us the ability of measuring the accuracy of our method. The results obtained using the scheme presented here, are in full agreement with other methods and solvers and the analytical solutions confirm the efficiency and effectiveness of the method. Where no other method is possible the modified trapezoidal rule is used for definite integrals, it also applies to approximating differential equations even a more general system of differential and algebraic equations and medical science [39]. It was observed however, that the accuracy of the method strictly depends on the step size $(h)$.

\section{Competing Interests}

The authors declared that no conflict of interest exist in this article.

\section{How to Cite this Article:}

J. Mamman and T. Aboiyar, "A Numerical Calculation of Arbitrary Integrals of Functions", Adv. J. Grad. Res., vol. 7, no. 1, pp. 11-17, Oct. 2019. doi:10.21467/ajgr.7.1.11-17

\section{References}

[1] I. Podlubny, "Geometric and physical interpretation of Fractional Integration and Fractional Differentiation," Fractional Calculus and Applied Analysis, vol. 5, no. 4, pp367-386. (2002) Math.CA/0110241

[2] I. Podlubny, \& R. Magin, \& I. Trymorush, "Niels Henrik Abel and the birth of fractional calculus," Fractional Calculus and Applied Analysis. (2017). 20

[3] I. Podlubny, "What Euler could further write, or the unnoticed big bang of the fractional calculus," Fractional Calculus and Applied Analysis. (2013). 16.

[4] I. Podlubny, \& M. Tavazoei, \& B. Vinagre, \& D. Xue, \& Y. Chen, \& M. Haeri, "A Special Issue in ISA Transactions Fractional Order Signals, Systems, and Controls: Theory and Application”. ISA Transactions (2018). 82. 1.

[5] T. S. Chow, "Fractional dynamics of interfaces between soft-nanoparticles and rough substrates," Physics Letter A, 342(1-2):148155. July (2005).

[6] R.T. Baillie, "Long memory processes and fractional integration in econometrics," J Econometrics, 73:5-59 (1996).

[7] R. L. Bagley, P. J. Torvik, "A theoretical basis for the application of fractional calculus to viscoelasticity," Journal Rheol. 27(3):201210 (1983).

[8] R. Panda, M. Dash, "Fractional generalized splines and signal processing,” Signal Process, 86:2340-2350 (2006). 
Mamman et al., Adv. J. Grad. Res.; Vol. 7, Issue 1, pp: 11-17, January 2020

[9] F. Mainardi, "Fractional calculus: some basic problems in continuum and statistical mechanics. In:Fractals and fractional calculus in continuum mechanics," New York: Springer, Verlag. p. 291-348 (1997).

[10] Q. Feng, A. Liu “Oscillation for a Class of Fractional Differential Equation,” Journal of Applied Mathematics and Physics 7, 14291439. (2019).

[11] K. Wang, \& S. Liu, "He's fractional derivative and its application for fractional Fornberg-Whitham equation," Thermal Science. 2016. 54-54. (2016).

[12] J. Shilpi and A. Praveen "On New Applications of Fractional Calculus," Boletim da Sociedade Paranaense de Matematica 37(3):113118 (2019).

[13] V. Tarasov, (2019). "On History of Mathematical Economics: Application of Fractional Calculus," Mathematics. 7. 509.

[14] D. Luo, \& J. Wang, \& M. Feckan, "Applying Fractional Calculus to Analyze Economic Growth Modelling," Journal of Applied Mathematics, Statistics and Informatics, (2018). 14. 25-36.

[15] C. Li, \& Y. Chen, \& J. Kurths, "Fractional calculus and its applications" Philosophical Transactions of The Royal Society A Mathematical Physical and Engineering Sciences. (2013). 371. 20130037.

[16] R. Hilfer "Applications of Fractional Calculus in Physics" Universität Mainz \& Universität Stuttgart, Germany. (2000) .

[17] A. Kochubei, \& Y. Kondratiev, "Growth Equation of the General Fractional Calculus" Mathematics, (2019). 7. 615.

[18] V. E. Tarasov, \& V. V. Tarasova, "Macroeconomic models with long dynamic memory: Fractional calculus approach," Applied Mathematics and Computation, Elsevier, vol. 338(C), pages 466-486. 2018.

[19] H. Sun, \& Y. Zhang, \& D. Baleanu, \& W. Chen, \& Y. Chen, "A new collection of real world applications of fractional calculus in science and engineering," Communications in Nonlinear Science and Numerical Simulation. (2018). 64.

[20] B. Datsko, \& I. Podlubny, \& Y. Povstenko, "Time-Fractional Diffusion-Wave Equation with Mass Absorption in a Sphere under Harmonic Impact,” Mathematics. (2019). 7. 433.

[21] R. Magin, \& B. Vinagre, \& I. Podlubny, "Can Cybernetics and Fractional Calculus Be Partners?: Searching for New Ways to Solve Complex Problems," IEEE Systems, Man, and Cybernetics Magazine. 4. 23-28. (2018).

[22] J. Machado, \& V. Kiryakova, "The Chronicles of Fractional Calculus". Fractional Calculus and Applied Analysis. 20(2), pp. 307336. (2017).

[23] J. Sabatier, \& C. Ionescu, \& J. Tar, \& M. J. Tenreiro, "New Challenges in Fractional Systems," Mathematical Problems in Engineering. (2013).

[24] R. Hilfer, and Y. Luchko, "Desiderata for fractional Derivatives and Integrals," Mathematics (2019) 7(2), 149.

[25] E. K. RobertoGarrapa, and P. Marina "Evaluation of fractional Integrals and Derivatives of elementary functions: Overview and Tutorial," Mathematics (2019) 7. 407.

[26] V. Kiryakova, "Use of fractional calculus to evaluate some improper integrals of special functions." AIP Conference Proceedings. (2017). 1910. 050012.

[27] P. Agarwal, "Fractional Integration of the Product of Two Multivariables H-Function and a General Class of Polynomials Praveen Agarwal," Springer Proceedings in Mathematics \& Statistics Volume 41, 2013, pp 359-374. (2013).

[28] Yuri Luchko (Eds.)," Basic Theory Berlin, Boston: De Gruyter. (pp. 111-126). (2019).

[29] M. J. Tenreiro \& V. Kiryakova, \& F. Mainardi, \& S. Momani, "FCAA-Round Table-ICFDA18," Fractional Calculus and Applied Analysis. 21. 1151-1155. (2018).

[30] F. Liu, \& M. Meerschaert, \& S. Momani, \& N. Leonenko, \& W. Chen, \& O. Agrawal, "Fractional Differential Equations" International Journal of Differential Equations. 2013.

[31] S. S. Uttam Ghosh, , D. Shantanu "Solution of System of Linear Fractional Differential Equations with Modified Derivative of Jumarie Type," American Journal of Mathematical Analysis. 2015; 3(3):72-84.

[32] M. Razzaghi, "A numerical scheme for problems in fractional calculus" ITM Web of Conferences. 20. 02001.

[33] V. Tarasov, \& S. Tarasova, "Probabilistic Interpretation of Kober Fractional Integral of Non-Integer Order," Progress in Fractional Differentiation and Applications. (2019). 5. 1-5.

[34] K. Diethelm, N. Ford, A. Freed, "Detailed error analysis for a fractional Adams method," Numerical Algorithms 36:31-52 May (2004).

[35] Z. Odibat, "Approximations of fractional integrals and Caputo fractional derivative," J Applied Mathematics and Computation, 178:527-533 (2006).

[36] J. H. Mathews, K. D. Fink, “Numerical Methods Using Mathlab”, Prentice-Hall, (2004).

[37] J. D. Daniel "Finite Difference Methods in Financial Engineering: A Partial Differential Equation Approach," Wiley Online Books.

[38] P. W. George Hornberger "Introduction to Finite Difference Methods for Partial Differential Equations," (2005) .

[39] M. M.Tai "A Mathematical model for the determination of total area under glucose tolerance and other metaboliccurves," Diabetes Care 17(2): 152-154 (1994).

Publish your books with AIJR publisher-

Publish with ISBN and DOI.

Publish Thesis/Dissertation as Monograph.

Publish Book Monograph.

Publish Edited Volume/ Book.

Publish Conference Proceedings

Retain full copyright of your books.

Submit your manuscript at books.aijr.org
Publish your research article in AIJR journals-

Online Submission and Tracking

Peer-Reviewed

$\checkmark$ Rapid decision

$\checkmark \quad$ Immediate Publication after acceptance

$\checkmark \quad$ Articles freely available online

Retain full copyright of your article.

Submit your article at journals.aijr.in 